\title{
Brillouin scattering effect in the multicore optical fiber applied to fiber optic shape sensing
}

\begin{abstract}
Anna Pytel, Agnieszka Kołakowska, Michalina Jóźwik, Łukasz Szostkiewicz, Zhisheng Yang, et al.
\end{abstract}

Anna Pytel, Agnieszka Kołakowska, Michalina Jóźwik, Łukasz Szostkiewicz, Zhisheng Yang, Barbara Wajnchold, Michał Murawski, Marek Napierała, Luc Thévenaz, Paweł Mergo, Tomasz Nasiłowski, "Brillouin scattering effect in the multicore optical fiber applied to fiber optic shape sensing," Proc. SPIE 10435, Technologies for Optical Countermeasures XIV, 104350N (5 October 2017); doi: 10.1117/12.2279528 


\title{
Brillouin scattering effect in the multicore optical fiber applied to fiber optic shape sensing
}

\author{
Anna Pytel ${ }^{1,2,{ }^{*}}$, Agnieszka Kołakowska ${ }^{1,2}$, Michalina Jóźwik ${ }^{1,2}$, tukasz Szostkiewicz ${ }^{1,2}$, Zhisheng Yang ${ }^{3}$, \\ Barbara Wajnchold ${ }^{4}$, Michał Murawski ${ }^{4}$, Marek Napierała ${ }^{1,2}$, Luc Thévenaz ${ }^{3}$, Paweł Mergo ${ }^{5}$, Tomasz \\ Nasiłowski ${ }^{1}$ \\ ${ }^{1}$ InPhoTech Sp. z o. o., 17 Slominskiego St. 31, 00-195 Warsaw , Poland \\ ${ }^{2}$ Faculty of Physics, Warsaw University of Technology, 75 Koszykowa St., 00-662 Warsaw, Poland \\ ${ }^{3}$ EPFL, Swiss Federal Institute of Technology, Institute of Electrical Engineering, SCI-STI-LT Station \\ 11, $\mathrm{CH}-1015$ Lausanne, Switzerland \\ ${ }^{4}$ Polish Centre For Photonics And Fibre Optics, 312 Rogoznica, 36-060 Glogow Malopolski, Poland \\ ${ }^{5}$ Laboratory of Optical Fiber Technology, Faculty of Chemistry, Maria Curie-Sklodowska University, \\ 5 M. Curie-Sklodowskiej Place, 20-031 Lublin, Poland \\ *apytel@inphotech.pl
}

\begin{abstract}
A shape sensor exploiting Brillouin scattering measurements in multicore fibers is presented. Based on previous reports ${ }^{1}$, the shape sensor's principle of operation is firstly described. The presented idea is realized through Brillouin Frequency Shift (BFS) measurements in the time domain along the entire multicore fiber. The BFS value is related to the strain value in each core and the differential inter-core strains lead to the bend radius and orientation. Authors present an experimental demonstration of the shape sensor using a 7-core microstructured optical fiber.
\end{abstract}

Keywords: Shape senor, Multicore optical fiber technology, Brillouin scattering in multicore optical fibers, Distributed sensing

\section{INTRODUCTION}

Distributed sensors utilizing the Brillouin scattering effect are widely used to measure temperature and $\operatorname{strain}^{2-4}$. Recently there is a huge interest in beating the range limits, improving spatial resolution and reducing the measurement time ${ }^{5}$. In the high-range systems used for distributed measurement of temperature and strain, standard optical fibers are most commonly applied ${ }^{2-5}$.

On the other hand, microstructured multicore optical fibers, which possess the diversity and possibility of changing the light propagation properties, are applied for various sensing applications, such as temperature ${ }^{6}$, strain $^{7}$ and bend measurements ${ }^{8}$. Additionally, multicore fibers are suitable for inscription of fiber Bragg gratings $(\mathrm{FBG})^{9}$, which can be useful for shape sensing ${ }^{10,11}$. Recently, observations of Brillouin scattering effect in multicore fibers were reported ${ }^{12}$ and the effect was successfully applied for shape sensing ${ }^{1}$. Unlike in previous report ${ }^{1}$, here authors used multicore fiber with air-hole isolation.

Shape sensing is a subject which has drawn much interest in the past few years. There are various optical fiber sensing architectures, but in general, shape sensing is possible if the exact bend radius, its location and orientation can be

Technologies for Optical Countermeasures XIV, edited by David H. Titterton, Robert J. Grasso,

Mark A. Richardson, Proc. of SPIE Vol. 10435, 104350N · @ 2017 SPIE .

CCC code: $0277-786 X / 17 / \$ 18 \cdot$ doi: $10.1117 / 12.2279528$ 
measured. To this end, authors take advantage of distributed sensing using Brillouin scattering in multicore optical fibers.

\section{PRINCIPLE}

As mentioned above, shape can be calculated once having information about a bend radius, orientation and location. The exact bend radius is deduced from the measurement of strain induced in lateral cores, which impacts on the BFS. Additionally, the comparison of the differential strain between cores enables to distinguish the bending orientation. The bending location is identified by performing an optical time-domain measurement, such as the Brillouin Optical Time Domain Analysis (BOTDA).

The presented sensor is based on a 7-core microstructured fiber (MCF7) designed for telecommunication applications (Fig. 1) ${ }^{13-15}$. The MCF7 structure consists of a central core and 6 lateral cores arranged hexagonally and it is optimized to ensure low attenuation and low optical cross-talk ${ }^{16}$ (less than $-30 \mathrm{~dB} @ 1550 \mathrm{~nm}$ ). The MCF7's cores diameter and doping is comparable with the ITU-T G.652 recommendations hence optical properties of each MCF7's core are similar as in the standard single-mode fibers.

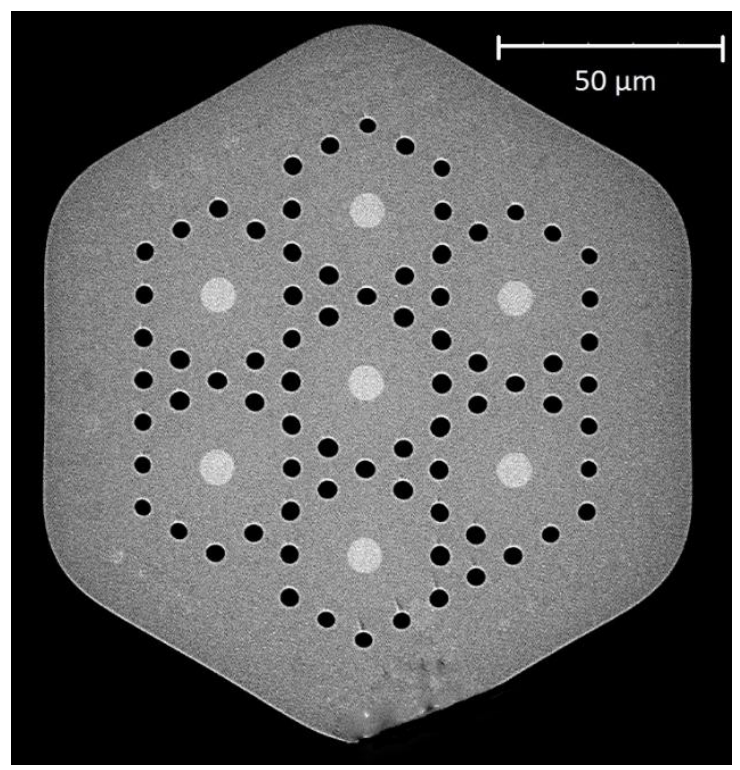

Fig. 1 Scanning electron microscope image of MCF7.

In distributed measurements based on the Brillouin scattering effect, backcoupled light with shifted frequency is observed. This frequency shift, called Brillouin Frequency Shift (BFS), is a value which is constant in unchanged external conditions for a given fiber and is defined as ${ }^{1,2}$

$$
v_{B}=\frac{2 n_{e f f} V_{a}}{\lambda}
$$

where $n_{e f f}$ is the effective refractive index, $V_{a}$ is the acoustic wave velocity and $\lambda$ is the incident wavelength.

For a bended fiber the BFS value in each core is different, as it depends on the induced strain, as shown in Fig. 2. Crosssection of the bended multicore fiber shows that each core localized in a bending plane will experience differential strains. Comparing to the central core, the strain experienced by the lateral core, which is on the inside of the bend, is negative, as this core is actually compressed. On the other side, the core outside of the bend experiences positive strain, as this core is actually elongated. The central core is located on the neutral bending line and therefore 
experiences no strain, providing a zero-bending reference value. This corresponds to the BFS presented in the graph (Fig. 2).

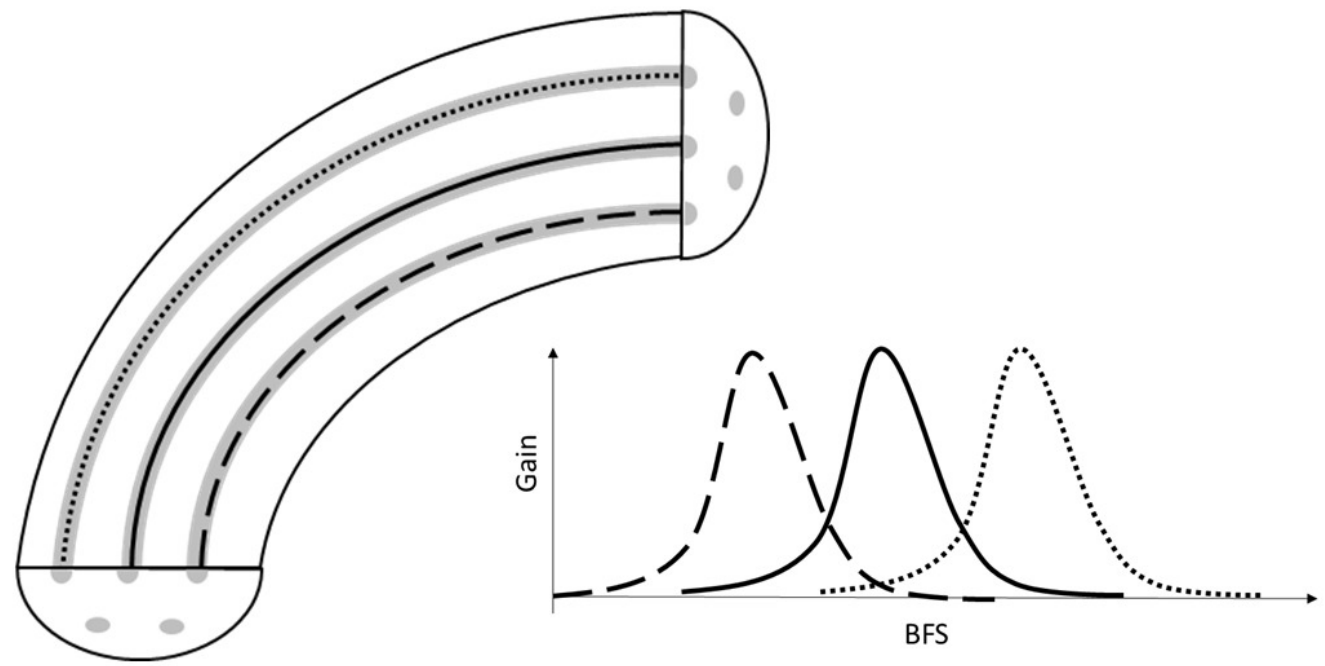

Fig. 2 BFS in the bended multicore fiber. On the left the cross-section along bended multicore fiber, on the right, the graph with corresponding BFS values for three cores is shown. BFS is smaller for the compressed core and greater for the elongated core.

Strain in an arbitrary lateral core depends on the bend radius and bend orientation, which can be expressed as ${ }^{1}$ :

$$
\varepsilon_{i}=-\frac{d_{i}}{R} \cos \left(\theta_{b}-\theta_{i}\right)
$$

where $\varepsilon_{i}$ is strain of the $\mathrm{i}$-th core, $d_{i}$ is the distance between the $\mathrm{i}$-th core and the center of the fiber, $R$ is the bend radius, $\theta_{b}$ is the bend orientation and $\theta_{i}$ is the angular position of the $\mathrm{i}$-th core. For the sake of clarity $d_{i}, \theta_{b} \theta_{i}$ are shown in Fig. 3.

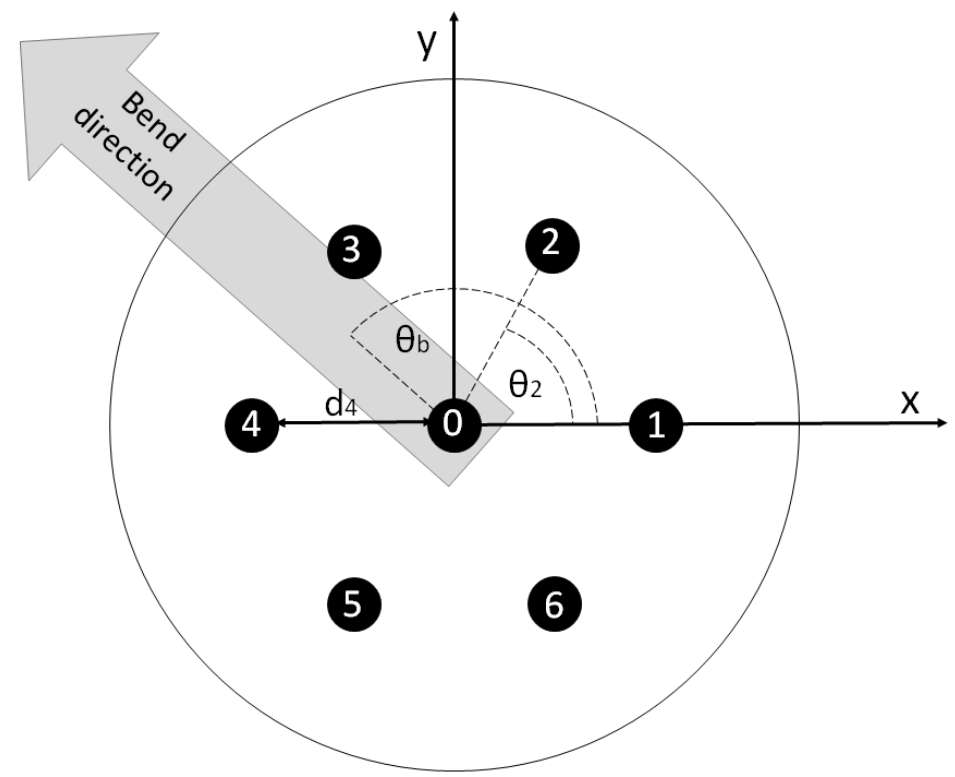

Fig. 3 The XY-plane cross-section of the bended fiber with outer cores (marked from 1 to 6 ) and central core (marked as 0 ). 
The BFS is linearly proportional to any signed strain variations. The proportionality constant is called strain sensitivity $\alpha$. Hence, the BFS can be expressed using the mentioned relation for strain

$$
\frac{\Delta v_{B i}}{v_{B}}=\varepsilon_{i} \alpha=-\frac{\alpha d_{i}}{R} \cos \left(\theta_{b}-\theta_{i}\right)
$$

where $\Delta v_{B i}$ is a variation of the BFS in the $\mathrm{i}$-th core and $v_{B}$ is an initial BFS value in reference conditions (in the case of the MCF7 the BFS for central core can be used). Based on this relation and acquiring BFS measurements for at least 3 lateral cores, it is possible to determine the bend radius and orientation at each point along the fiber in the 3D space 1,17

\section{EXPERIMENTAL SETUP}

The BFS in each core of the MCF7 was measured using the set-up presented in Fig. 4 using Brillouin Optical Time Domain Analysis (BOTDA). The optical-time domain method enables to distinguish BFS for each point along the fiber and hence strain localization along the fiber. The tested MCF7 sample was $26 \mathrm{~m}$ long. Both ends of the MCF7 were spliced to tapered multicore fiber connectors $(T M C)^{18}$, which enable light injection and detection into and from each core separately.

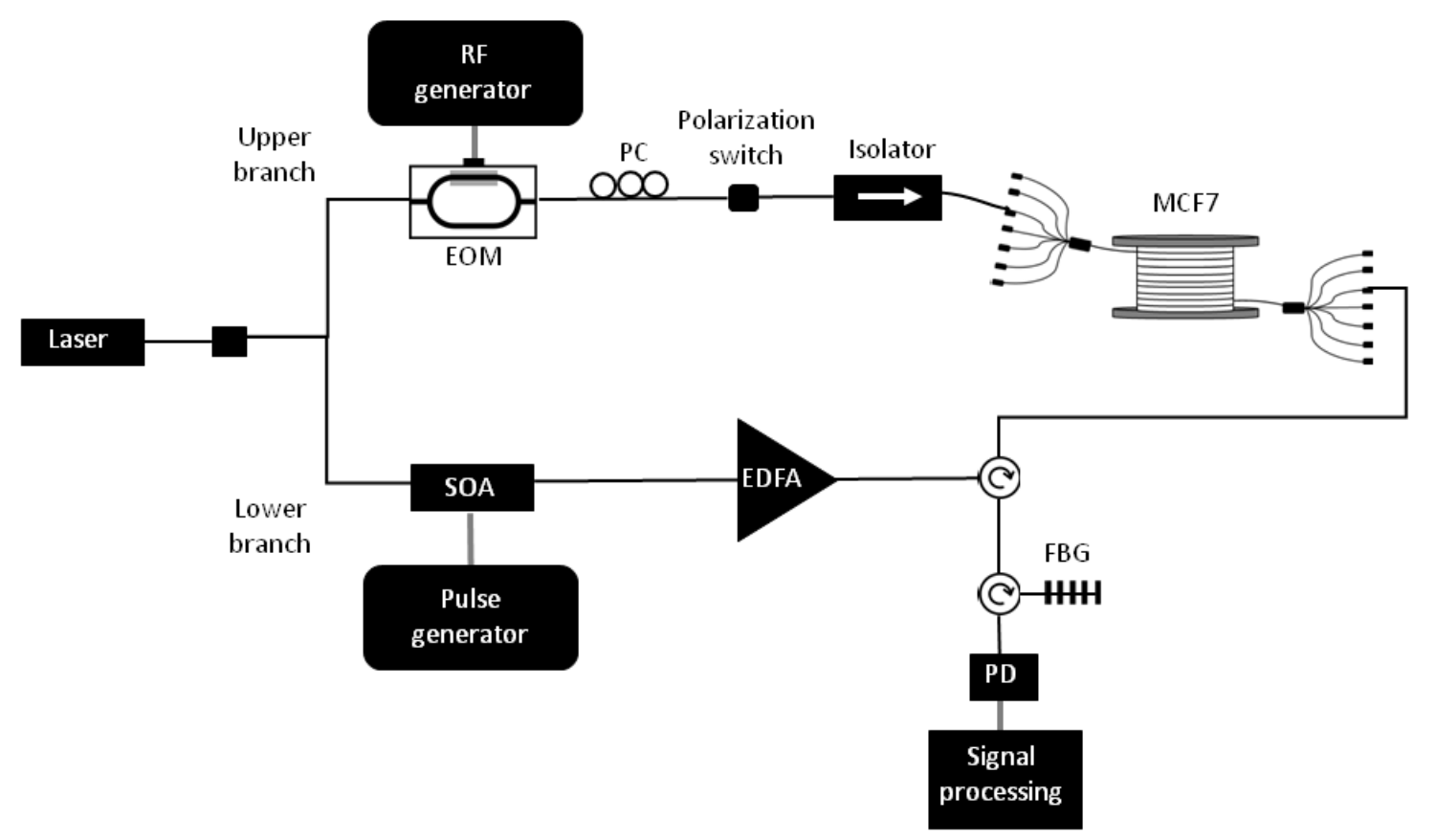

Fig. 5 Experimental setup: EOM: electro-optic intensity modulators, PC: polarization controller, SOA: semiconductor optical amplifier, EDFA: erbium-doped fiber amplifier, FBG: fiber Bragg grating, PD: photodetector.

The measurement is performed with $1 \mathrm{~m}$ spatial resolution using a standard distributed feedback laser operating at $1551 \mathrm{~nm}$ as a light source. Light from the laser is divided into two branches. In the upper branch a probe signal with two sideband frequencies is created. Sideband frequencies are generated using an electro-optic intensity modulator driven by tunable radio frequencies from a RF generator ${ }^{2}$. Then the signal propagates through a polarization switch where the polarization state of the probe signal can be switched between orthogonal states during the measurement. Polarization switching is necessary to avoid the effect of polarization-induced fading and it is performed by summing up the response for two orthogonal polarizations. Then, through the TMC, the probe signal is launched into a specific core of the MCF7. In the lower branch the pump signal is generated. Light from the laser propagates through a 
semiconductor optical amplifier where light pulses are shaped. Next, pulses are amplified using an erbium-doped fiber amplifier, and the pump signal goes via a circulator through the TMC to the tested MCF7. The probe signal and the light backcoupled in the MCF7 propagate to the receiver unit where one sideband frequency is filtered out using a fiber Bragg grating (FBG). Afterwards the signal is detected on the photodetector (PD) and analyzed to distinguish BFS at each location along the fiber.

\section{EXPERIMENTAL DEMONSTRATION}

BOTDA provides a measurement of the Brillouin Gain Spectrum (BGS) which is a 3-dimensional plot representing the probe signal gain for each frequency shift and each location along the tested fiber. The maximum gain of the probe signal corresponds to the situation when the difference between the probe signal and the pump signal is equal to the BFS. In Fig. 6 the Brillouin Gain Spectra for the central core and one of the lateral cores are shown.
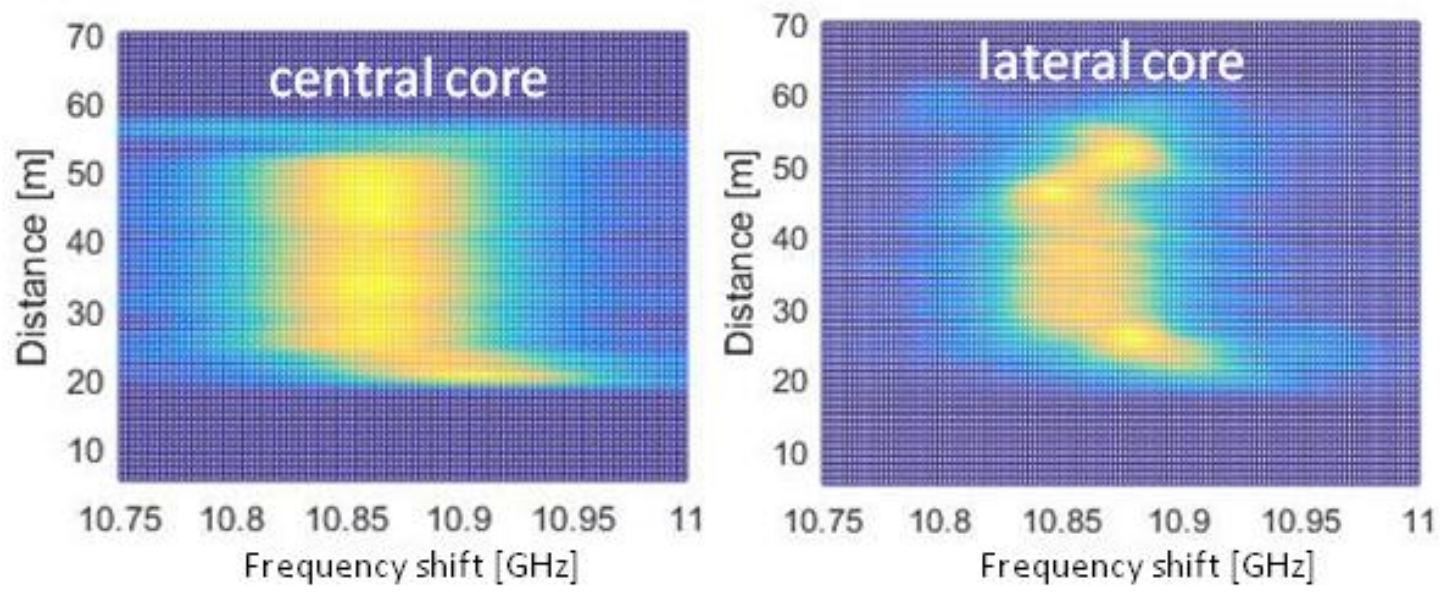

Fig. 6 Brillouin gain spectrum for central core (graph on the left) and one of the lateral cores (graph on the right). X-axis represents the frequency shift, which is equal to the frequency difference for the probe and pump signals, Y-axis represents the distance along the fiber. The MCF7 fiber is localized from the $25^{\text {th }} \mathrm{m}$ till $51^{\text {st }} \mathrm{m}$ of the measured optical path. The probe signal gain is represented in a color scale, where maximum is colored in orange and minimum in blue.

To introduce a specific shape of the MCF7, the fiber was coiled on a spool. That means that fiber was bended with a constant radius and randomly twisted. Experimental results were consistent with expectations, since in the central core of the MCF7 the measured BFS was constant along the whole fiber. In the same time, in the lateral core BFS varied along the fiber. The BFS variation in the lateral core was influenced by the random twist of the fiber. The observed core was once in the inside of the bend and once in the outside, that is, it was elongated and compressed alternatively. These variations of BFS along the MCF7 are clearly visible in Fig. 7. 


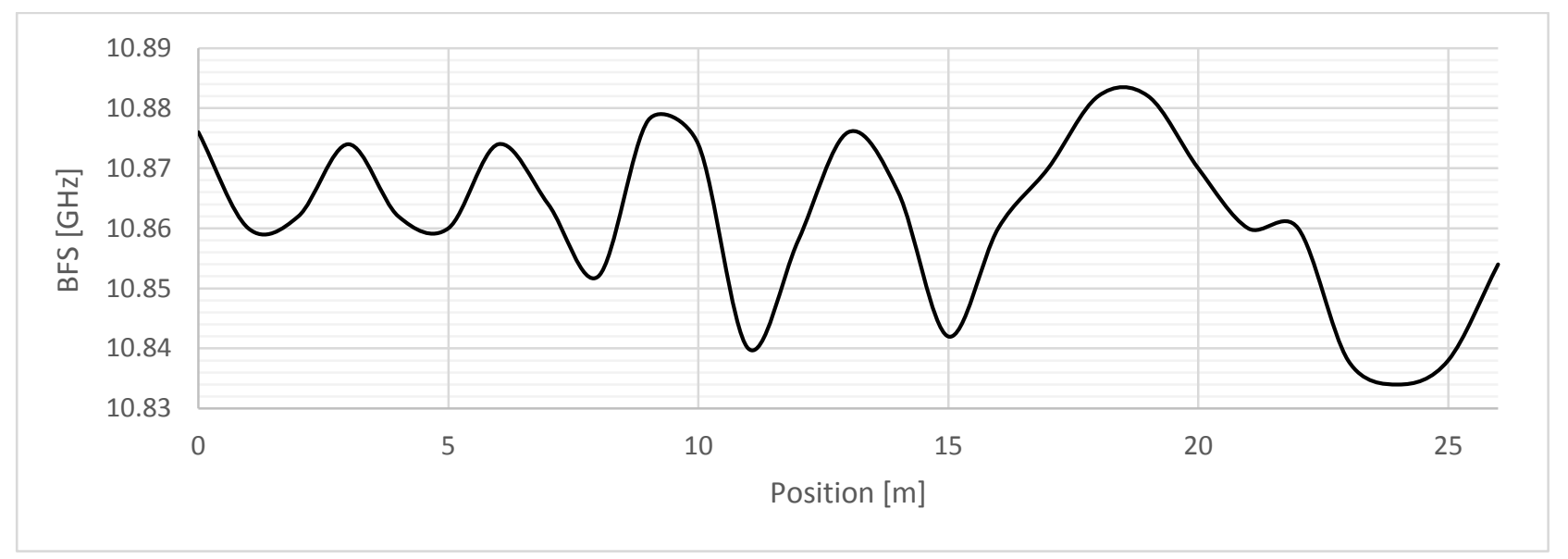

Fig. 7 Brillouin frequency shift variations for one of the lateral cores along the $26 \mathrm{~m}$-long MCF7.

\section{CONCLUSIONS}

Authors discussed the idea of shape sensing based on Brillouin Frequency Shift measurement in a multicore fiber, firstly reported by Z. Zhao at al. ${ }^{1}$. According to this idea, the BFS values measured at a given point for at least 3 lateral cores carry enough information to determine the bend radius and its orientation. This theory was confronted with the experimental demonstration, where the Brillouin gain spectrum along a $26 \mathrm{~m}$-long 7-core microstructured fiber was measured. The results for the central core and one of the lateral cores are presented. BFS variations for the lateral core related to twists and bend along the MCF7 confirm that shape sensing in the proposed configuration is possible.

Presented results give promising conclusions for the future. After improving the spatial resolution of the bend localization and insertion losses of the sensing element, it will be possible to measure shape with high accuracy.

\section{ACKNOWLEDGMENTS}

This research was partially supported by the Foundation for Polish Science within the TEAM-TECH program as a part of the "NODUS" project co-financed by the European Union under the European Regional Development Fund, as well as by the National Centre for Research and Development within the research projects PBS1/B3/12/2012.

\section{REFERENCES}

[1] Zhao, Z., Soto, M. A., Tang, M., Thévenaz, L., "Distributed shape sensing using Brillouin scattering in multi-core fibers," Opt. Express 24(22), 25211-25223 (2016).

[2] Nikles, M., Thevenaz, L., Robert, P. A., "Brillouin gain spectrum characterization in single-mode optical fibers," J. Light. Technol. 15(10), 1842-1851 (1997).

[3] Soto, M. A., Thévenaz, L., "Modeling and evaluating the performance of Brillouin distributed optical fiber sensors," Opt. Express 21(25), 31347-31366 (2013).

[4] Thévenaz, L., "Brillouin distributed time-domain sensing in optical fibers: state of the art and perspectives," Front. Optoelectron. China 3(1), 13-21 (2010).

[5] Motil, A., Bergman, A., Tur, M., "[INVITED] State of the art of Brillouin fiber-optic distributed sensing," Opt. Laser Technol. 78, 81-103 (2016). 
[6] Reimlinger, M., Colalillo, A., Coompson, J., Wynne, R., "Multicore photonic crystal fiber force meters," Proc. SPIE 7981, 79814K (2011).

[7] Ziołowicz, A., Bieńkowska, B., Budnicki, D., Jóźwik, M., Ostrowski, Ł., Murawski, M., Pytel, A., Tenderenda, T., Wójcik, G., Szostkiewicz, Ł., Makara, M., Poturaj, K., Mergo, P., Nasiłowski, T., "Supermode interference in dualcore hole-assisted fiber for sensing," Proc. SPIE 9816, 98160E (2015).

[8] Napierała, M., Szostkiewicz, Ł., Szymański, M., Mergo, P., Jaroszewicz, L. R., Nasiłowski, T., "Concept of all-fiber bend sensor based on photonic crystal fibers with asymmetric air-hole structure," Proc. SPIE 8794, Fifth European Workshop on Optical Fibre Sensors, 87941V (2013).

[9] Stępień, K., Słowikowska, M., Tenderenda, T., Murawski, M., Szymański, M., Szostkiewicz, Ł., Becker, M., Rothhardt, M., Bartelt, H., Mergo, P., Jaroszewicz, L. R., Nasiłowski, T., "Fiber Bragg gratings in hole-assisted multicore fiber for space division multiplexing," Opt. Lett. 39(12), 3571 (2014).

[10] Duncan, R. G., Froggatt, M. E., Kreger, S. T., Seeley, R. J., Gifford, D. K., Sang, A. K., Wolfe, M. S., “High-accuracy fiber-optic shape sensing," Proc. SPIE 6530, 65301S (2007).

[11] Flockhart, G. M. H., MacPherson, W. N., Barton, J. S., Jones, J. D. C., Zhang, L., Bennion, I., "Two-axis bend measurement with Bragg gratings in multicore optical fiber," Opt. Lett. 28(6), 387-389 (2003).

[12] Mizuno, Y., Hayashi, N., Tanaka, H., Wada, Y., Nakamura, K., "Brillouin scattering in multi-core optical fibers for sensing applications," Sci. Rep. 5(1), 1138 (2015).

[13] Ziołowicz, A., Szymanski, M., Szostkiewicz, Ł., Tenderenda, T., Napierała, M., Murawski, M., Hołdyński, Z., Ostrowski, Ł., Mergo, P., Poturaj, K., Makara, M., Słowikowski, M., Pawlik, K., Stańczyk, T., Stępień, K., Wysokiński, K., Broczkowska, M., Nasiłowski, T., "Hole-assisted multicore optical fiber for next generation telecom transmission systems," Appl. Phys. Lett. 105(8), 81106 (2014).

[14] Napierała, M., Ziołowicz, A., Pytel, A., Szostkiewicz, Ł., Murawski, M., Tenderenda, T., Ostrowski, Ł., Szymański, M., Jóźwik, M., Filipowicz, M., Makara, M., Poturaj, K., Mergo, P., Nasiłowski, T., "Multicore optical fibres for next generation telecommunication transmission systems and components," Photonics Lett. Pol. 7(3) (2015).

[15] Pytel, A., Napierała, M., Szostkiewicz, Ł., Ostrowski, Ł., Murawski, M., Mergo, P., Nasiłowski, T., “Optical power 1 x 7 splitter based on multicore fiber technology," Opt. Fiber Technol. 37, 1-5 (2017).

[16] Szostkiewicz, Ł., Napierała, M., Ziołowicz, A., Pytel, A., Tenderenda, T., Nasiłowski, T., "Cross talk analysis in multicore optical fibers by supermode theory," Opt. Lett. 41(16), 3759-3762 (2016).

[17] Moore, J. P., Rogge, M. D., "Shape sensing using multi-core fiber optic cable and parametric curve solutions," Opt. Express 20(3), 2967-297 (2012).

[18] Zhu, B., Taunay, T. F., Yan, M. F., Fini, J. M., Fishteyn, M., Monberg, E. M., Dimarcello, F. V., "Seven-core multicore fiber transmissions for passive optical network," Opt. Express 18(11), 11117-11122 (2010). 